Vol. 2 Issue 2, Feb.2012, pp. 372-375

\title{
A Novel Machine Vision System for Radish Crop Quality Monitoring based on Leaf Inspection
}

\author{
Amina Khatra ${ }^{1}$ and Maninder Kaur ${ }^{2}$ \\ ${ }^{1}$ (ECE Deptt., Doaba Institute of Engineering \& Technology, Kharar, Punjab INDIA) \\ ${ }^{2}$ (ECE Deptt., Doaba Institute of Engineering \& Technology, Kharar, Punjab INDIA)
}

\begin{abstract}
The crop of radish is very often infected by a disease that leaves spots of brown, gray or off-white colors on the radish leaves in winter. Scientifically, this disease is known as cercospora leaf spot or cercospora cruciferarum. It's a kind of fungus that often kills young seedlings. The fungus spreads by air and can also infect radish plants when splashed onto leaves during a rainfall as the radish grows up down the soil as stem and leaves come out of the soil. Therefore, it is important to monitor the leaf at regular intervals so as to keep track on quality of growing radish crop. In the presented paper, a novel machine vision system has been proposed that visually inspects the leaves coming out of the soil and based on spots on leaves, it determines the nature of fungus and its depth into the radish stem. The size of the fungus, color depth and location and locus of the fungus on leaves gives an accurate determination of crop quality under the soil. In the presented thesis work, the image of the crop leaves are taken by a good quality color camera and processed for getting a gray colored and segmented image depending upon the nature and size of the fungus. A criterion is set for acceptable and rejects crop quality based on the fungus level.
\end{abstract}

\section{INTRODUCTION}

Radish is one of the vegetables that can be grown in all agro-ecological regions throughout the year. Radish leaves differ in color, shape, size and texture from normal to infected ones [4]. As the radish crop is grown up under the soil, it is very much important to observe the quality of the crop by inspecting the same from top. And that can be checked by monitoring the leaves coming out of the soil. In the presented work, the radish leaves are examined for their shape, size, color and presence of any fungus or spot. Also, the leaf's shape is checked for its shape with respect to the fact that it is not bitten by any animal [2]. A bitten leaf will therefore be an incomplete leaf along with some marks of the larva on leaves; and thereby infecting the crop. Leaves are the integral part of any under soil crop and the crop gets its required natural nutrients through the leaf only. Therefore, the morphology of the leaf gives a fair idea of its crop quality.

One major disease Downy mildew is caused by the fungus Peronospora parasitica. It overwinters in roots from diseased plants. The disease appears as small yellow leaf spots that later turn brown with bluish-black lace-like markings. Under wet conditions, a white downy mold develops in the

underside of the leaf [3]. The leaf spots render the radishes unmarketable.
Another disease White rust causes pale dusty spots to develop on the lower surface of the radish leaves. The causal fungus overwinters on residue from diseased plants. Dusty spores from the leaf spots can be spread long distances by wind and short distances by the movement of workers and tools in the field. The appearance of spots on the radish leaves destroys its marketability [1].

In the presented paper, the visual inspection of radish leave is divided into following steps:

1. Image Acquisition

2. Thresholding

3. Segmentation

4. Feature Extraction

5. Result Comparison

\section{IMAGE ACQUISITION}

In the proposed thesis work, the radish leaves images are acquired by using a CCD camera of approx. 3 M-Pixel resolution in 24-bits color resolution. The images are then transferred to PC and represented in MATLAB software. Here, it is to be noted that the proposed system is not to identify or visually inspect each and every radish leaf, but rather to identify at least one infected leaf from the mass that may lead to infect the entire crop.

The key idea in the presented work is to develop a novel technique for detection of any disease/infection from the leaves in radish crop that suffers at a higher rate than other underground vegetables. The main problem in radish leaves image capturing arises on account of cover area. For, it is suggested to install no. of close circuit CCD cameras to cover small areas at critical positions in the field like in the centre, corners and periphery. After capturing the image, the radish leaves images are brought under image processing techniques to identify the growth and type of infections. Fig. 1 shows the group of images taken by CCD camera from the field.
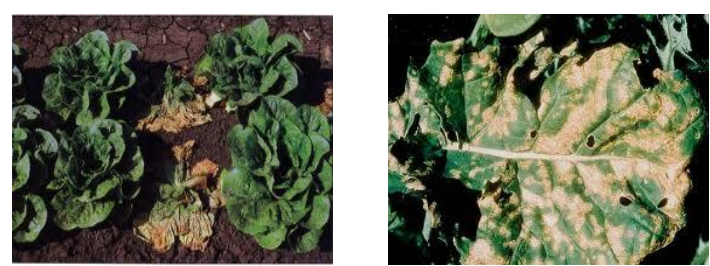
Vol. 2 Issue 2, Feb.2012, pp. 372-375
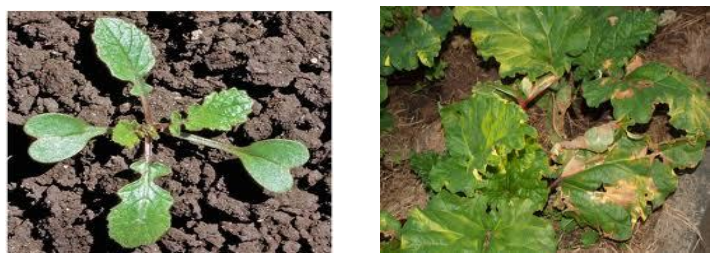

Fig. 1

\section{THRESHOLDING}

Acquired color image is now converted to gray scale color scheme using MATLAB command rgb2gray. Local and global thresholding approaches may be utilized to produce a binary image from the gray scale image. In the present work, a median filter of $3 \times 3$ kernel is applied and then a thresholding algorithm based on Otsu method is applied (global thresholding) [6]. Fig. 1 and 2 shows the original image taken from the field in 24-bits color format. Fig. 3 is the image after converting the same into gray shade format. Median filter is applied to remove the salt and pepper noise which is normally present in the crop field images. Median filtering is similar to using an averaging filter, in that each output pixel is set to an average of the pixel values in the neighborhood of the corresponding input pixel. However, with median filtering, the value of an output pixel is determined by the median of the neighborhood pixels, rather than the mean. The median is much less sensitive than the mean to extreme values (called outliers). Median filtering is therefore better able to remove these outliers without reducing the sharpness of the image.

The results after applying the median filter are shown in fig. 4. When Otsu algorithm is applied over the filtered image, a binary image as shown in Fig. 5 is obtained.

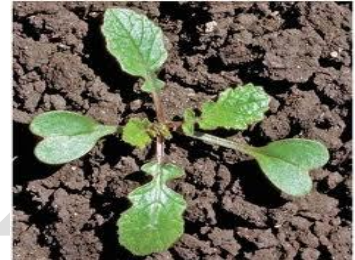

Fig. 2

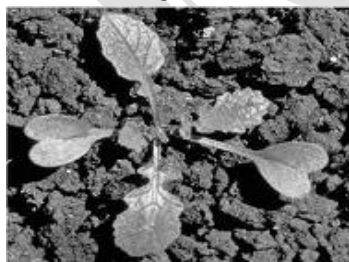

Fig. 4

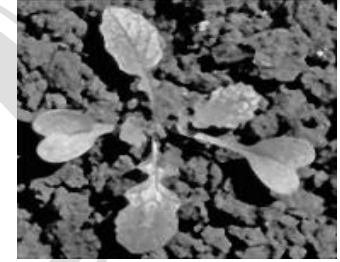

Fig. 3

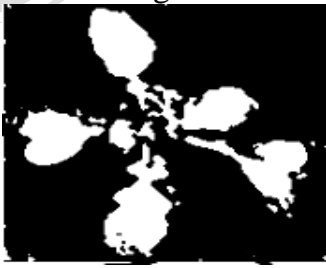

Fig. 5

\section{SEGMENTATION}

The threshold image is now segmented based on bwlabel algorithm in MATLAB. Fig. 5 is taken as input image for segmentation. Below are the figures after segmentation using 8-connectivity of pixels and bwlabel function in MATLAB software.
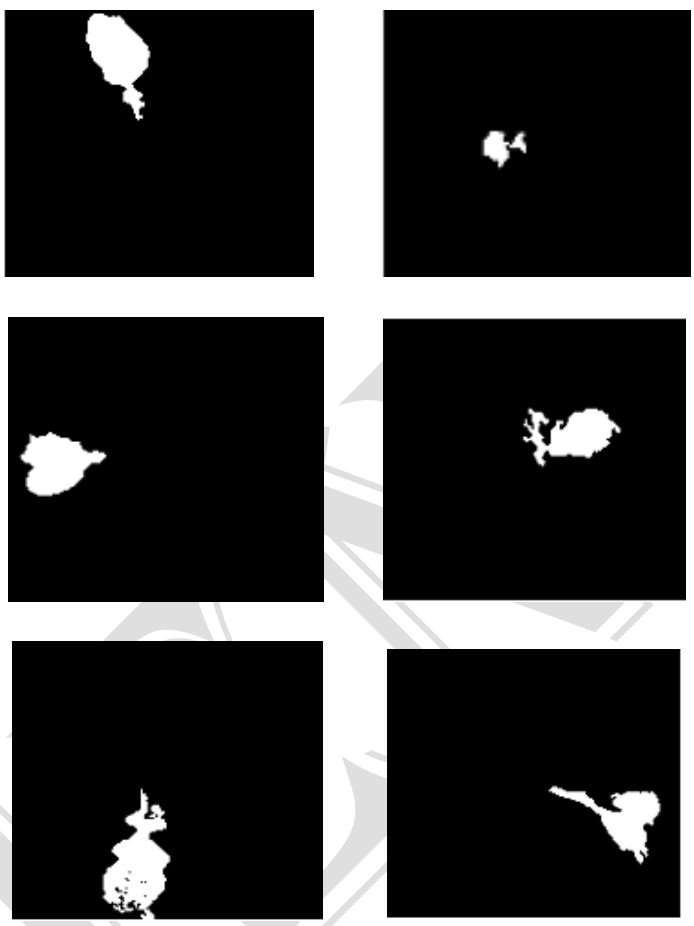

Fig. 6

Small leaf portions based on connected pixels basis can be left out as they do not contribute much in visual inspection of radish crop. Another segmentation based on color is also employed in the presented work. Color based segmentation suggest the decoloring of the leaf due to any fungus present in the crop. Fig. 7 shows the radish leaf with very high fungus level.

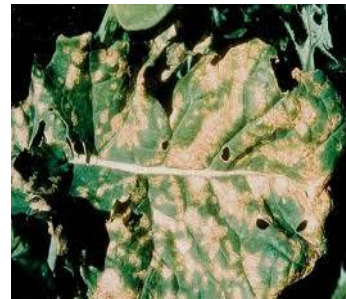

Fig. 7

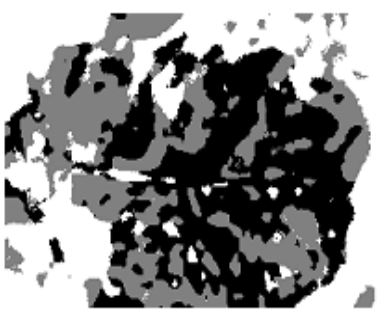

Fig. 8
Fig. 8 shows the segmentation of leaf based on color segmentation using K-means clustering approach. Clustering is a way to separate groups of objects. K-means clustering treats each object as having a location in space. It finds partitions such that objects within each cluster are as close to each other as possible, and as far from objects in other clusters as possible.

\section{FEATURE EXTRACTION}

In the presented paper work, following observations are supposed to be monitored: 


\section{Amina Khatra and Maninder Kaur / IOSR Journal of Engineering (IOSRJEN) www.iosrjen.org \\ ISSN : 2250-3021}

Vol. 2 Issue 2, Feb.2012, pp. 372-375

1. Leaf Morphology

2. Leaf Area, perimeter

3. Completeness of the leaf

4. De-coloring of leaf due to fungus or disease

Leaf Morphology of the leaf suggests and compares the leaf under test with the standard or reference image of the leaf. Leaf morphology comparison between standard and leaf under test is approximate and not exact. However, the normalization of leaf under test with respect to size is must before comparison.

Leaf Area and perimeter suggest the size of the leaf after normalization of the leaf size with respect to standard one.

Completeness of the leaf with respect to its morphology suggests that if any part of the leaf is bitten by an animal, then, that part of the field may be infected by the animal's larva transferred on the leaf. That larva may penetrate into the crop and may infect the same and the nearby plants as well.

De-coloring of the leaf indicates the presence of fungus and that may be due to any of the disease like cercospora leaf spot or cercospora cruciferarum. The de-coloring of the leaf may be identified either by spot analysis or by color based segmentation as shown in fig. 7 and 8.

\section{RESULTS}

Following table shows the area and perimeter of the segmented leaves using the regionprops function in MATLAB software.

\begin{tabular}{|c|c|c|c|}
\hline S. No. & $\begin{array}{c}\text { Area } \\
\text { (sq. Pixels) }\end{array}$ & $\begin{array}{c}\text { Perimeter } \\
\text { (Pixels Unit) }\end{array}$ \\
\hline 1 & & 471 & 192 \\
\hline 2 & & 485 & 217 \\
\hline 3 & & 497 & 239 \\
\hline 4 & & 201 & 78 \\
\hline 5 & & 543 & 234 \\
\hline
\end{tabular}

Mean

Perimeter $\quad 196$

Area 452

However, if the leaf at s. no. 4 is left out and mean and standard deviations are recomputed, then we get:

$\begin{array}{lll} & \text { Mean } & \text { Standard Deviation } \\ \text { Perimeter } & 220 & 16 \\ \text { Area } & 502 & 25\end{array}$

Comparison of above calculation suggest that the normal spreads of area and perimeter are 114 and 54 respectively i.e. towards higher side. However, if we leave the leaf at sr. no. 4 , we get the spread of area and perimeter as 25 and 16 , that are very much controlled for a normal plant leaves. Therefore, on this basis it can be suggested that if the spread is very high, this indicates that the leaves are not of regular size and shapes and there may be a possibility of animal byte. Also, if we observe the fig. 8, that has been segmented based on color using K-means clustering, we get the ratio of area of gray to black pixels i.e. ratio of green part to the fungus part and it comes out to be more than $40 \%$. This shows that the leaf is infected by the fungus and may penetrate into the crop down the soil.

\section{Conclusion}

This study suggests that the visual inspection of radish leaves gives fair idea of the crop quality regarding any animal byte, fungus and growth. Particularly, when the crop grows under the soil and direct monitoring of the crop is not possible. Therefore, a correlation is to be made between the properties of leaves above soil to that of the vegetable under the soil. Visual inspection proposes totally non-destructive testing (NDT) of the crop quality based on the leaf condition. However, the soil surface images may also be taken into consideration, if a correlation is possible between the surface shades and crop quality.

\section{ACKNOWLEDGEMENTS}

We are thankful to Mr. Vikas Goel, Sr. Project Manager, CDAC, Mohali, Punjab for his valuable guidance and continuous support throughout the entire thesis work.

From the table above, the mean and standard deviation of area and perimeters comes out to be as follows: 


\section{Amina Khatra and Maninder Kaur / IOSR Journal of Engineering (IOSRJEN) \\ www.iosrjen.org \\ ISSN : 2250-3021}

Vol. 2 Issue 2, Feb.2012, pp. 372-375

\section{REFERENCES}

[1] S.S. Abu-Naser, K. A. Kashkash and M. Fayyad [2008] "Developing an Expert System for Plant Disease Diagnosis, Journal of Artificial Intelligence"

[2] Mohammad Ei -Helly, Ahmed Rafea, Salwa Ei - Gamal And Reda Abd Ei Whab[2004] Integrating Diagnostic Expert System With Image Processing Via Loosely Coupled Technique, Central Laboratory for Agricultural Expert System(CLAES).

[3] Zhi-Chun Huang, Patrick P. K. Chan, Wing. W. Y. Ng, Daniel S. Yeung, "Content based image retrieval using COLOR moment and gabor texture feature", Proceedings of 9th international conference on machine learning and cybernetics,Qingdao, pp-719724,11-14 July 2010
[4] R. Brunelli and O. Mich, "Histograms Analysis for Image Retrieval," Pattern Recognition, Vol.34, No.8, pp16251637,2001

[5] P. S. Suhasini, Dr. K. Sriramkrishna, Dr. I. V. Muralikrishna "CBIR using Color histogram processing", Journal of theoretical and applied information technology,pp-116-122,2009

[6] J. Huang, S. R. Kumar, M. Mitra, W. J. Zhu, and R. Zabih, "Image indexing using Color correlograms" ,IEEE Int. Conf. Computer Vision and Pattern Recognition, San Juan, Puerto Rico, Jun. 1997, pp.762- 768.

[7] Jau-Ling Shih \& Ling-Hwei Chen, "Color image retrieval based on primitives of Color moments". 